ELECTRONIC RESEARCH ANNOUNCEMENTS OF THE AMERICAN MATHEMATICAL SOCIETY

Volume 5, Pages 47-54 (April 8, 1999)

S $1079-6762(99) 00059-1$

\title{
METRIC MINIMIZING SURFACES
}

\author{
ANTON PETRUNIN
}

(Communicated by Dmitri Burago)

\begin{abstract}
Consider a two-dimensional surface in an Alexandrov space of curvature bounded above by $k$. Assume that this surface does not admit contracting deformations (a particular case of such surfaces is formed by area minimizing surfaces). Then this surface inherits the upper curvature bound, that is, this surface has also curvature bounded above by $k$, with respect to the intrinsic metric induced from its ambient space.
\end{abstract}

\section{INTRODUCTION}

Let $M$ be a Riemannian manifold and $S$ a smooth surface in $M$. One can write a Gaussian formula for the curvature of $S$,

$$
K_{S}=K_{M}+K_{G},
$$

where $K_{S}$ is the curvature of $S, K_{M}$ the curvature of $M$ in the same direction, and $K_{G}$ the Gaussian curvature in the same direction. Two important corollaries arise. First, if $M$ has positive curvature and $S$ is a convex hypersurface, then the intrinsic metric on $S$ has positive curvature. Second, if $M$ has negative curvature and $S$ is a two-dimensional saddle surface (i.e., the principal curvatures for any normal direction, do not have the same sign at any point), then the intrinsic metric on $S$ has negative curvature.

All notions in these corollaries have natural sense in Alexandrov's geometry (one can define convex surface, saddle surface (see below) and curvature bounded below and above for general length-metric space). Therefore one can ask if the generalization of such results is true for Alexandrov spaces. Both of these problems are open so far.

Here we give a proof of a special case of the second problem. Roughly speaking, we prove that upper bound on curvature is inherited by a surface in an Alexandrov space with curvature $\leq k$, if this surface does not admit a contracting deformation (this is stronger than just being saddle; see below). The proof uses the well-known idea of approximating our metric by a polyhedral one, but there is one funny point below, that is the place where we consider an approximation of a metric on a surface by a metric on a graph (it was a real surprise for me that this worked).

I thank the anonymous referee for invaluable help in making this paper readable, as well as one of the editors for bringing the thesis of C. Mese to my attention.

Received by the editors September 14, 1998.

1991 Mathematics Subject Classification. Primary 53C21.

The main part of this note was prepared when the author had a postdoctoral fellowship at MSRI (Berkeley).

(C)1999 American Mathematical Society 


\section{Definitions AND NOtATiOn}

Let $X, Y$ and $Z$ be compact metric spaces.

1.1. Notation. We will write $X-\epsilon \leq Y$ or $X \leq Y+\epsilon$ if there is a mapping $f: X \rightarrow Y$ such that $\left|x x^{\prime}\right|_{X} \leq\left|f(x) f\left(x^{\prime}\right)\right|_{Y}+\epsilon$.

It is easy to see that this is a transitive relation in the following sense:

If $X-\epsilon \leq Y$ and $Y-\epsilon^{\prime} \leq Z$ then $X-\epsilon-\epsilon^{\prime} \leq Z$.

1.2. Remark. It is easy to see that in this notation if $X$ is $\epsilon$-close to $Y$ in the Gromov-Hausdorff sense (see [GLP]), then $X-2 \epsilon \leq Y$ and $Y-2 \epsilon \leq X$. On the other hand, from the old folklore lemma which says that if $X \geq Y$ and $Y \geq X$, then $X$ is isometric to $Y$ (a careful proof can be found in [P, 1.2]), it is easy to see that if $X_{n}-\epsilon_{n} \leq Y$ and $Y-\epsilon_{n} \leq X_{n}$ and $\epsilon_{n} \rightarrow 0$, then $X_{n} \stackrel{G H}{\rightarrow} Y$. Therefore if one defines the metric

$$
d^{\prime}(X, Y)=\min \{\epsilon ; X-\epsilon \leq Y \text { and } Y-\epsilon \leq X\},
$$

then this new metric will define the same topology on the set of compact metric spaces as the standard Gromov-Hausdorff metric.

1.3. Now $M$ is a metric space.

Definition. A function $f: U \subset M \rightarrow R$ is called convex if for any geodesic $\gamma \subset U$ with length-parameter, $f \circ \gamma$ is convex.

Definition. We denote by $D$ a closed disk in $R^{2}$. A continuous mapping $s: D \rightarrow$ $M$ is called saddle if for any convex function $f$ on $M$ and any subset $U \subset D$

$$
\sup _{x \in U} f(x)=\sup _{x \in \partial U} f(x) .
$$

Remark. The definition of saddle surfaces in $R^{3}$ used by Shefel is that a plane cannot cut a cap from the surface. If one applies the definition given above to affine functions, it is easily seen that the two definitions are equivalent for the case $M=R^{3}$.

Notation. For a mapping $s: D \rightarrow M$ define a pseudometric on $D$ by $|x y|=$ $|s(x) s(y)|_{M}$. Let $|x y|_{s}, x, y \in D$, be the infimum of $|* *|$-length of curves in $D$ connecting $x$ and $y$. We will call $|* *|_{s}$ the pull-back metric (generally speaking, it is a pseudometric, i.e. it is possible to have $|x y|_{s}=0$ for $x \neq y$, but we call it a metric anyway).

Definition. A continuous mapping $s: D \rightarrow M$ is called metric minimizing if the pull-back metric is compact and there is no mapping $s^{\prime}: D \rightarrow M$ such that $\left.\left.s^{\prime}\right|_{\partial D} \equiv s\right|_{\partial D},\left|x x^{\prime}\right|_{s^{\prime}} \leq\left|x x^{\prime}\right|_{s}$ for any two points $x, x^{\prime} \in D$, and the inequality is strict for at least one pair.

In some sense it is the weakest "minimal"-like property one could express in terms of the intrinsic metric of a surface.

\section{Results AND PROOFs}

2.1. Conjecture. (Probably due to Shefel.) Let $M$ be an Alexandrov space with curvature $\leq 0$. Then any saddle mapping $s: D \rightarrow M$ gives a pull-back metric on $D$ with curvature $\leq 0$. 
This conjecture was proved by S. Shefel ([She1] and [She2]) in two special cases: first for $M=R^{2}$, and second for $M=R^{3}$ (the last one with the additional assumption that $s$ describe a surface which is locally a graph of a function). Aleksandrov [A] proved that the upper bound on curvature is inherited by ruled surfaces in an Alexandrov space with curvature $\leq k$. C. Mese in her thesis proved this conjecture for minimal surfaces in Alexandrov spaces (see $[\mathrm{M}]$ ).

Now look what I can do:

2.2. Theorem. Let $M$ be a locally compact Alexandrov space with curvature $\leq k$ or a complete Alexandrov space with curvature $\leq k \leq 0$. Then any metric minimizing mapping $s: D \rightarrow M$ gives a pull-back metric on $D$ with curvature $\leq k$.

Let us prove first that this is really a special case of the Conjecture.

2.3. Proposition. Any metric minimizing mapping in an Alexandrov space with curvature $\leq k$ is a saddle mapping.

Remark. The author does not know a counterexample to the conjecture that any saddle mapping in an Alexandrov space with curvature $\leq k$ and rectifiable $s \circ \partial D$ is metric minimizing. Therefore one can consider this Theorem as a way to approach the Conjecture.

Proof of the Proposition. Let $M$ be an Alexandrov space with curvature $\leq k$ and $s: D \rightarrow M$ be metric minimizing. Assume there is a convex function $f$ such that $f \circ s$ admits a strict local maximum at $C, C$ is compact and $|C \partial D|_{s}>0$.

Direct application of Sharafutdinov retraction [Sha] for level surfaces of $f$ in a neighborhood of $s(C)$ (this is an abstraction of deformation along gradient curves in the smooth case) gives us a $|* *|_{s}$-nonexpanding deformation of $s$ in an arbitrary small neighborhood of $C$.

Remark. The condition that our space be an Alexandrov space with curvature $\leq 0$ is not necessary. In order to apply Sharafutdinov retraction it is enough that our space have the first variation formula, i.e. one can define angle between two geodesics which start from the same point such that the standard first variation formula holds for distance functions.

2.4. Proof of the Theorem. Sections $2.4-2.8$ will deal with the locally compact case; in Section 2.9 the necessary extra work is done for complete negatively curved Alexandrov spaces.

For the convenience of the reader I will consider only the case $k=0$. The general case requires only technical modifications.

Without loss of generality one can assume that $\partial D$ is rectifiable in the pull-back metric and $\operatorname{Im}(s)$ is inside some $R_{0}$-domain (see $[\mathrm{N}]$ ).

Let us construct a "formal" triangulation $\Gamma$ in $\left(D,|* *|_{s}\right)$ whose 1-skeleton with intrinsic metric is $\epsilon$-GH-close to $\left(D,|* *|_{s}\right)$ and no side of any triangle is longer than $\epsilon$. "Formal" means that the sides in the triangulation can have overlapping segments. Therefore triangles could be degenerate like the one shown in Figure 1.

Let us denote by $\Gamma_{0}, \Gamma_{1}$ the 0 - and 1-skeleton of $\Gamma$.

2.5. Construction of $\Gamma$. (If you do believe in this, skip to 2.6.)

Let us consider a finite $\nu$-net $\left\{a_{i}\right\}$ in $\left(D,|* *|_{s}\right)$. Consider the minimal closed curve $\gamma \subset\left(D,|* *|_{s}\right)$ which surrounds all $a_{i}$ 's, and let us denote by $\operatorname{Conv}\left\{a_{i}\right\}$ the set of points surrounded by $\gamma$. 


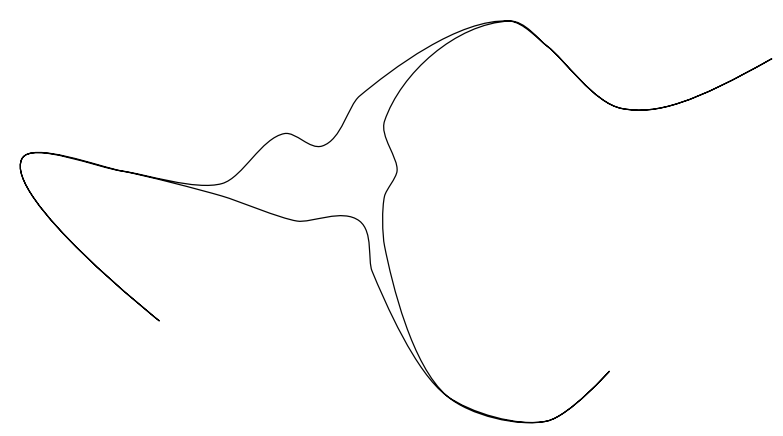

Figure 1
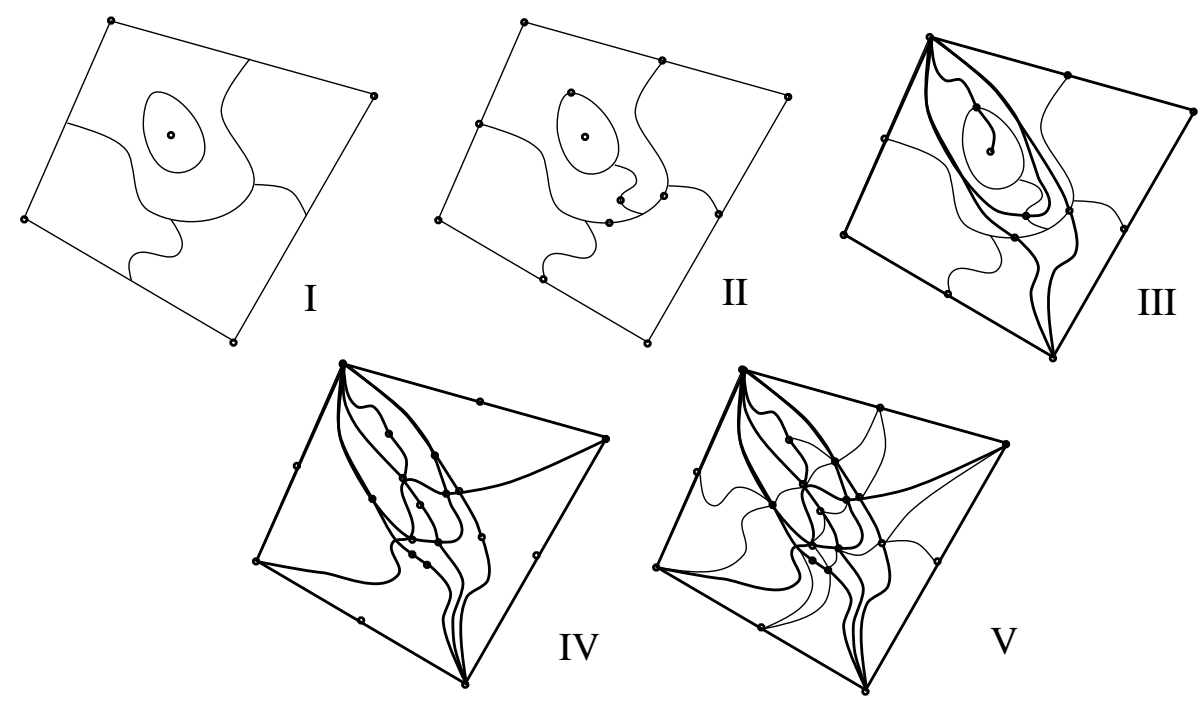

FigURE 2

First let us subdivide $\operatorname{Conv}\left\{a_{i}\right\}$ into polyhedra with small diameters and perimeters. The steps of this subdivision are illustrated in Figure 2, in which subfigure I shows the division into $C_{i}$ with a net of 5 points.

For every $a_{i}$ consider the set

$$
C_{i}=\left\{x \in \operatorname{Conv}\left\{a_{i}\right\} ;\left|x a_{i}\right| \leq\left|x a_{j}\right|\right\} .
$$

We can add to $\left\{a_{i}\right\}$ finitely many points on $\partial \operatorname{Conv}\left\{a_{i}\right\}$ to meet the following property: if $C_{j} \cap \partial \operatorname{Conv}\left\{a_{i}\right\} \neq \emptyset$, then $a_{j} \in \partial \operatorname{Conv}\left\{a_{i}\right\}$.

Let us consider a fundamental domain in the universal covering $\tilde{C}_{i}$ of $C_{i}$,

$$
D_{i}=\left\{x \in \widetilde{C}_{i} ;\left|x \tilde{a}_{i}\right| \leq\left|x \gamma\left(\tilde{a}_{i}\right)\right| \text { for any } \gamma \in \pi_{1}\left(C_{i}\right)\right\} .
$$

Mapping $D_{i}$ back to $D$, we obtain a map on $\operatorname{Conv}\left\{a_{i}\right\}$ such that every "country" is a disk. On every segment-border or point-border between countries choose a point ("customs point"), including borders with the same country. (See II in Figure 2.) Connect every "customs point" by a minimal geodesic with the "capital" $\left(a_{i}\right)$ of every adjacent country (if it is a border between a country and itself, one has to 
consider two or more connections depending on how many pieces of this country meet at this customs point). (See III in Figure 2.) Therefore we have cut $\operatorname{Conv}\left\{a_{i}\right\}$ into hexagons and quadrangles with perimeter $\leq 6 \nu$ and diameter $\leq 5 \nu$. (The number of vertices of a polygon is even because, when we are going along its perimeter, "capitals" and "customs points" will alternate. It is easy to see that the number of capitals in one polygon is at most 3 , since otherwise there must be some other customs inside the polygon; therefore the total number of vertices is $\leq 6$.)

It is easy to see that the "customs points" can be chosen so that the constructed hexagons and quadrangles cover all of $\operatorname{Conv}\left\{a_{i}\right\}$.

Now let us remove the borders and connect each pair of the $a_{i}$ 's by a minimal geodesic; we can easily do it to meet the rule: every two geodesics have either empty intersection, or one-point intersection or one-segment intersection (including the already constructed geodesics between capitals and customs points). This insures that we obtain a finite graph. (See IV in Figure 2.)

We obtain a partition of $\operatorname{Conv}\left\{a_{i}\right\}$ into polyhedra of perimeter $\leq 6 \nu$ and diameter $\leq 5 \nu$. Indeed, every polygon of our graph is a result of finitely many cuts of a hexagon or quadrangle along a geodesic, and the perimeter as well as the diameter do not increase at any such step.

Now let us subdivide every polygon into triangles with the same vertices. (See $\mathrm{V}$ in Figure 2.)

We obtain a triangulation of $\operatorname{Conv}\left\{a_{i}\right\}$ in $\left(D,|* *|_{s}\right)$. It is easy to show that the 1-skeleton of this triangulation is $8 \nu$-close to $\left(D,|* *|_{s}\right)$ in the sense of the $d^{\prime}$ metric (see 1.2).

Indeed, by mapping each point to a closest $a_{i}$ we have $\left(D,|* *|_{s}\right)-2 \nu \leq \Gamma_{1}$. Now the distance between any point of $\Gamma_{1}$ (in its length-metric) and a closest element of $\left\{a_{i}\right\}$ is less than $4 \nu$ (for $3 \nu / 2$ we can get from any point to the perimeter of a polyhedron, for another $3 \nu / 2$ we can traverse the perimeter of the hexagon or quadrangle, and after another $\nu$ we get a point from the $\nu$-net). Therefore by mapping every point of $\Gamma_{1}$ to a closest (in the intrinsic metric of $\Gamma_{1}$ ) point from the $\nu$-net we obtain that $\Gamma_{1}-8 \nu \leq\left(D,|* *|_{s}\right)$. Therefore we obtain the needed triangulation $\Gamma$ for sufficiently small $\nu=\nu\left(D,|* *|_{s}, \epsilon\right)$ (see 1.2).

2.6. One can naturally define the boundary of $\Gamma(\partial \Gamma)$.

Let us consider the image $s\left(\Gamma_{0}\right) \subset M$ and connect vertices which are connected in $\Gamma$ by geodesics in $M$. Call the new graph $\dot{\Gamma}$. It is easy to see that $\dot{\Gamma} \leq \Gamma_{1}$.

Now let us consider a graph $\ddot{\Gamma}$ with the same combinatorial type, which minimizes the total length of edges in the set of graphs such that every edge is less than or equal to the corresponding edge of $\dot{\Gamma}$, and with the same boundary as $\dot{\Gamma}$.

Applying Arzelà-Ascoli arguments we conclude that such a graph exists. Obviously we have $\ddot{\Gamma} \leq \dot{\Gamma}$.

2.7. Now let us consider a polyhedral metric on the triangulation, with edge lengths given by those in $\ddot{\Gamma}$. Simply consider for any triangle in $\ddot{\Gamma}$ (which represent a triangle in the triangulation) a model triangle in $R^{2}$ and glue a triangulation from them. Let it be $C(\ddot{\Gamma})$. It is easy to see that $C(\ddot{\Gamma})-2 \epsilon \leq \ddot{\Gamma}$ because from the construction every side of every triangle is not more than $\epsilon$.

It is easy to construct a nonexpanding mapping of $m: C(\ddot{\Gamma}) \rightarrow M$ (by [R]) such that $\left.m\right|_{\ddot{\Gamma}}=$ id. We can consider the pull-back metric on $C(\ddot{\Gamma})$. 
Therefore,

$$
\left(C(\ddot{\Gamma}),|* *|_{m}\right)-2 \epsilon \leq C(\ddot{\Gamma})-2 \epsilon \leq \ddot{\Gamma} \leq \dot{\Gamma} \leq \Gamma_{1} \leq\left(D,|* *|_{s}\right)+2 \epsilon .
$$

Hence

$$
\left(C(\ddot{\Gamma}),|* *|_{m}\right) \leq C(\ddot{\Gamma}) \leq\left(D,|* *|_{s}\right)+4 \epsilon .
$$

As $\epsilon \rightarrow 0$ (again applying Arzelà-Ascoli arguments) we obtain a nonexpanding mapping $\left(D,|* *|_{s}\right) \rightarrow M$ with the same boundary values as $s$. From the metric minimizing property of $s$, this new mapping has exactly the same pull-back metric, i.e.,

$$
\lim _{\epsilon \rightarrow 0}\left(C(\ddot{\Gamma}),|* *|_{m}\right)=\left(D,|* *|_{s}\right) .
$$

From the inequality above,

$$
\lim _{\epsilon \rightarrow 0} C(\ddot{\Gamma})=\left(D,|* *|_{s}\right) .
$$

Therefore, the following Proposition finishes the proof.

2.8. Proposition. $C(\ddot{\Gamma})$ has nonpositive curvature.

Proof. Indeed, assume the contrary; then there is a vertex in $C(\ddot{\Gamma})$ with sum of angles $<2 \pi$. Let us consider the space of directions $\overline{\Omega_{p}(M)}$ at this point. It follows from [N, Theorem 1] that it is a CAT(1) space, and from the comparison inequality we obtain that the directions of the edges at $p$ form a broken geodesic with total length $l<2 \pi$. By using Reshetniak's theorem (see [R] or [N, Corollary 2]) we obtain that there is a convex domain in $C \subset S^{2}$ with perimeter $l$ admitting a nonexpanding mapping $r: C \rightarrow \overline{\Omega_{p}(M)}$ such that $\operatorname{Im} \partial C$ is our broken geodesic. Therefore, there is a direction $\omega \in \overline{\Omega_{p}(M)}$ which has angle $<\pi / 2$ with the direction of every edge at $p$ (it is the image of the "center" of $C$ ). By moving $p$ along a curve in a direction close to $\omega$ we reduce every edge of $\ddot{\Gamma}$, a contradiction.

2.9. What to do when $M$ is only complete, but $k \leq 0$. For spaces which are not locally compact the very same proof works, but we cannot apply Arzelà-Ascoli arguments. There are two places where we did it: the first is the existence of $\ddot{\Gamma}$ (2.6), and the second is the existence of the limit $\ddot{\Gamma}$ as $\epsilon \rightarrow 0$ (2.7).

The existence of $\ddot{\Gamma}$ can be proved in the following way:

Let $p_{i}(\dot{\Gamma})$ be a sequence of graphs with the same boundary as $\dot{\Gamma}$, such that the total length of edges of $p_{i}(\dot{\Gamma})$ goes to infimum. Passing to a subsequence if necessary one can insure that the length of each edge converges as $i \rightarrow \infty$. Assume it is not a Cauchy sequence. Then there are two subsequences, $p_{n_{i}}(\dot{\Gamma})$ and $p_{m_{i}}(\dot{\Gamma})$ such that for some $\epsilon>0$,

$$
\max _{x \in \Gamma_{0}}\left|p_{n_{i}}(x) p_{m_{i}}(x)\right|>\epsilon .
$$

Let us take $q_{i}(x), x \in \Gamma_{0}$, to be the midpoint of $p_{n_{i}}(x) p_{m_{i}}(x)$. Then for any two points $x, y \in \dot{\Gamma}_{0}$ the Bruhat-Tits inequality gives

$$
2\left|q_{i}(x) q_{i}(y)\right| \leq\left|p_{n_{i}}(x) p_{n_{i}}(y)\right|+\left|p_{m_{i}}(x) p_{m_{i}}(y)\right|
$$

and for some edge the difference between the right and left sides of the inequality is greater than some positive number which does not depend on $i$. 
Indeed, let $d(x)=\left|p_{n_{i}}(x) p_{m_{i}}(x)\right|$. Then since the length of each edge converges, the last statement is a trivial corollary of the following inequality for $x, y \in \dot{\Gamma}_{0}$ :

$$
2\left|q_{i}(x) q_{i}(y)\right|^{2}+\frac{1}{2}|d(x)-d(y)|^{2} \leq\left|p_{n_{i}}(x) p_{n_{i}}(y)\right|^{2}+\left|p_{m_{i}}(x) p_{m_{i}}(y)\right|^{2} .
$$

This inequality (as well as the one above) is obvious for a plane quadrangle, and from triangle comparison it is true for Alexandrov spaces with nonpositive curvature, a contradiction.

The fact that $\lim _{\epsilon \rightarrow 0}\left(C(\ddot{\Gamma}),|* *|_{m}\right)=\left(D,|* *|_{s}\right)$, needed in 2.7, can be obtained in a similar way:

First note that for each $\ddot{\Gamma}=\ddot{\Gamma}(\epsilon)$ one can construct a mapping $f_{\epsilon}: D \rightarrow M$ such that:

(i) $\left|f_{\epsilon}(x) f_{\epsilon}(y)\right| \leq|x y|_{s}+\epsilon$;

(ii) $\left.\left.f_{\epsilon}\right|_{\partial D} \equiv s\right|_{\partial D}$.

(One simply needs to take $\left.\left.f_{\epsilon}\right|_{\partial D} \equiv s\right|_{\partial D}$ and then send each point in $D \backslash \partial D$ to the vertex of $\ddot{\Gamma}$ corresponding to the closest point $a_{i}$ of the $\nu$-net; see 2.5.)

Now, for $\epsilon \rightarrow 0$, consider the set of all possible GH-limits of $D$ with the extrinsic pseudometric induced from $f_{\epsilon}$ (i.e., $|x y|=\left|f_{\epsilon}(x) f_{\epsilon}(y)\right|$; here $f_{\epsilon}$ has the above properties).

Obviously each such limit $X \leq\left(D,|* *|_{s}\right)$. Consider a minimal element $X_{0}$ in this set, and take a sequence $f_{\epsilon_{n}}\left(\epsilon_{n} \rightarrow 0\right)$ which corresponds to $X_{0}$. Then the same arguments as above show that $f_{\epsilon_{n}}$ is a Cauchy sequence. Therefore as a limit we have a mapping $f: D \rightarrow M$ such that $|f(x) f(y)| \leq|x y|_{s}$, but from the definition of pull-back metric we then have $|x y|_{f} \leq|x y|_{s}$, and from the metric minimizing property we have $|x y|_{f}=|x y|_{s}$. Since for appropriately chosen $X_{0}$ we can assume

$$
\left(D,|* *|_{f}\right) \leq \lim _{\epsilon \rightarrow 0}\left(C(\ddot{\Gamma}),|* *|_{m}\right) \leq\left(D,|* *|_{s}\right),
$$

we have

$$
\lim _{\epsilon \rightarrow 0}\left(C(\ddot{\Gamma}),|* *|_{m}\right)=\left(D,|* *|_{s}\right) .
$$

2.10. Final remark. It is really strange that the sign of $k$ comes in the game in such a place. If one tried to repeat 2.9 for $k>0$, then some positive term would appear in the Bruhat-Tits inequality, which is very small but which will poison all the fun. Direct application of the above proof would still give convergence if our surface were in addition hodograph minimizing, in the sense of the following definition:

Definition. A metric minimizing mapping $s: D \rightarrow M$ is called hodograph minimizing if in addition there is no mapping $s^{\prime}: D \rightarrow M$, such that $\left.\left.s^{\prime}\right|_{\partial D} \equiv s\right|_{\partial D}$, $\left|x x^{\prime}\right|_{s^{\prime}} \leq\left|x x^{\prime}\right|_{s}$ for any two points $x, x^{\prime} \in \partial D$, and if the inequality is strict for at least one pair.

\section{REFERENCES}

[A] A.D. Aleksandrov, Ruled surfaces in metric spaces, Vestnik LGU Ser. Mat., Mech., Astr. 1957, vyp. 1, 5-26. (Russian) MR 19:59a

[GLP] M. Gromov, Structures métriques pour les variétés riemanniennes, J. Lafontaine and P. Pansu, eds., CEDIC, Paris, 1981. MR 85e:53051

[M] C. Mese, The curvature of minimal surfaces in singular spaces, to appear in Comm. Anal. Geom.

[N] I. Nikolaev, The tangent cone of an Aleksandrov space of curvature $\leq K$, Manuscr. Math. 86 (1995), No. 2, 137-147. MR 95m:53062 
[P] A. Petrunin, Parallel transportation for Alexandrov space with curvature bounded below, Geom. Funct. Anal. 8 (1998), No. 1, 123-148. MR 98j:53048

[R] Yu.G. Reshetniak, Non-expansive maps in a space of curvature no greater than $K$, Sibirskii Mat. Zh. 9 (1968), 918-928; English transl., Siberian Math. J. 9 (1968), 683-689. MR 39:6235

[Sha] V.A. Sharafutdinov, The radius of injectivity of a complete open manifold of nonnegative curvature, Doklady Akad. Nauk SSSR 231 (1976), No. 1, 46-48. MR 56:9459

[She1] S.Z. Shefel(Šefel' $)$, On saddle surfaces bounded by a rectifiable curve, Doklady Akad. Nauk SSSR 162 (1965), 294-296; English transl. in Soviet Math. Dokl. 6 (1965). MR 31:3945

[She2] S.Z. Shefel, On intrinsic geometry of saddle surfaces, Sibirsk. Mat. Zh. 5 (1964), 13821396. (Russian) MR 30:5232

Max-Planck-Institut für Mathematik in DEN NAturwissenschaften, InSElstrasse 2226, D-04103 Leipzig, Germany

E-mail address: petrunin@mailhost.mis.mpg.de 